\title{
Crónica del II Congreso Nacional de la Asociación de Mujeres en el Sector Público, celebrado el día 17 de noviembre de 2020
}

\author{
Chronicle of the II National Congress of the Association \\ of Women in the Public Sector, held on the $17^{\text {th }}$ \\ November 2020
}

\author{
VIRTUdES IGLESIAS MARTÍNEZ \\ Inspectora de Servicio del Ministerio de Industria, \\ Comercio y Turismo. \\ Vocal de la Junta Directiva de la Asociación de Mujeres \\ en el Sector Público. \\ Vocal de la Comisión de Igualdad de FEDECA. \\ PILAR MADRID YAGÜE \\ Doctora en Derecho. \\ Letrada de la Administración de la Seguridad Social. \\ Presidenta de la Comisión de Igualdad de Fedeca. \\ Miembro de la Asociación de Mujeres en el Sector Público.
}

La Asociación de Mujeres en el Sector Público celebró el día 17 de noviembre de 2020 su II Congreso Nacional, que este año se celebró en formato virtual lo que facilitó que aproximadamente 800 personas siguieran las ponencias programadas.

Abrió el Congreso la presidenta de la Asociación, Concepción Campos Acuña, que, tras dar la bienvenida a los asistentes, destacó que, en un año marcado por la COVID 19, se perciben los primeros síntomas de la crudeza de una crisis social que es especialmente preocupante en el caso de las mujeres y jóvenes, tal y como se concluye en los estudios realizados, como el reciente encuentro de los bancos centrales de Europa, Estados Unidos e Inglaterra o la encuesta realizada por la propia Asociación de Mujeres en la que se puso de manifiesto la doble penalización que ha sufrido la mujer durante esta pandemia.

El congreso fue inaugurado oficialmente por la Presidenta del Consejo de Estado, María Teresa Fernández de la Vega, y por la Ministra de Política Territorial y Función Pública, Carolina Darias San Sebastián.

La Presidenta del Consejo de Estado comenzó su intervención con el agradecimiento por poder participar en este II Congreso que pretende denunciar los retos existentes, 
pero también avanzar en la búsqueda de soluciones. Destacó que, pese a los avances, se mantiene en todas las áreas el predominio del estatus creado por el modelo masculino y excluyente en el espacio del poder y de la toma de decisiones. Estos avances, en opinión de Fernández de la Vega, son lentos e incompletos, por lo que se hace preciso dar un impulso a la agenda de la igualdad en el ámbito de lo público con dos objetivos a su juicio de carácter urgente: desmontar el carácter patriarcal del Estado y combatir el sesgo masculino de la lógica neoliberal, porque igualdad y espacio público van de la mano. Por lo que se hace necesario girar hacia un modelo de sociedad más inclusivo.

Seguidamente intervino la Ministra Carolina Darias San Sebastián señalando que en los últimos años se han producido importantes avances en materia de igualdad entre hombres y mujeres. Sin embargo, tal y como pone de manifiesto el Informe de la Igualdad de Género que aprobó la Comisión Europea en el año 2019, la desigualdad en los países de la UE sigue existiendo en muchísimos ámbitos. También en el ámbito de las Administraciones Públicas se reproducen las mismas dinámicas, roles y estereotipos que en otras organizaciones, pero en menor medida que en el resto. Atendiendo a los datos en el ámbito de la AGE el 51,4 \% son mujeres el 48,6 \% son hombres, pero el mayor porcentaje de mujeres se encuadran en los grupos C1 y C2. De los puestos correspondientes a los niveles más altos de la Administración solo el 42,4 \% de los niveles 30 están ocupados por mujeres, el 39,7 \% corresponden a nivel 29 y el $46 \%$ a nivel 28.

La Ministra destacó el valor estratégico de la igualdad para alcanzar una sociedad más justa, representando una "política palanca" de carácter transversal que impulsa la transformación de nuestras administraciones, que permite adoptar soluciones innovadoras, transformadoras de una administración que, como la sociedad, es cada vez más liquida y en la que no caben brechas digitales, territoriales ni, mucho menos, brechas de género. La igualdad de género posibilita atraer y retener talento.

Ma Ángeles Durán de las Heras, Profesora de Investigación ad honorem del CSIC, comenzó su intervención con el tema "Las mujeres en la administración pública y el teletrabajo" refiriendo algunas vivencias de carácter personal para ilustrar cómo ha sido la evolución del papel de la mujer en las actividades de carácter público y en la sociedad en general. Manifestó que, según la ley, la mujer tiene abiertas las puertas para su entrada en la Administración, pero en la práctica es un poco más complicado, no tanto porque lo impida el propio sistema de la Administración sino porque las mujeres llevan una carga extra de trabajo no remunerado que se realiza para la familia. En su opinión, hay que pedirle que se abran las puertas aún más, pero también hay que conseguir que en la opinión pública y en la conducta de nuestros inmediatos deje de considerarse que la mujer es la depositaria del cuidado. Concluyó afirmando que o se reparte el cuidado o las posibilidades de competir de la mujer son menores que la de los hombres.

Susana de la Sierra Morón, profesora de Derecho Administrativo de la Universidad de Castilla-La Mancha, "Las mujeres en el entorno digital: algunas consideraciones sobre la Carta de Derechos Digitales", hizo hincapié en los resultados de las encuestas de teletrabajo que arrojan datos demoledores. A modo de ejemplo, cita los resultados obtenidos en el ámbito 
universitario que concluyen que el teletrabajo ha servido para que muchos hombres hayan aprovechado para ampliar sus carreras, mientras que para las mujeres ha sido una trampa ya que la conciliación no ha sido tal. Para la profesora, el presente y el futuro son digitales y en las administraciones se ha de actuar no solo con leyes sino también con políticas públicas que ayuden a paliar las brechas digitales, en particular la brecha de género, entre otras.

Así mismo, expuso el estudio de ONTSI en el que se analiza cómo han avanzado las tecnologías en los últimos 12 años y cómo han impactado en las mujeres en relación con los hombres. Se detecta que los indicadores son peores en las mujeres y se insta a mejorar las políticas públicas, por ejemplo, despertando la vocación STEM en las niñas. Para concluir su intervención, pasó a desarrollar brevemente el proceso de elaboración del borrador de la Carta de Derechos Digitales que se encuentra en proceso de consulta pública hasta el 4 de diciembre de 2020. Destacó que la igualdad es un valor superior del ordenamiento jurídico y en la Carta se proyecta con ese carácter de valor superior, apareciendo en distintos apartados de la misma, además de considerar que se ha de promover la perspectiva de género en los procesos de transformación digital.

Ana de Miguel Álvarez, profesora de la Universidad Rey Juan Carlos, "El mito de la libre elección y la carrera profesional de las mujeres: cómo no retroceder en igualdad", profundizó en la idea de que la relación con el espacio público como forma de realización personal, no ya de autonomía económica, es un tema en el que se puede rastrear desde que surge el movimiento feminista: "no es este el mundo que queremos". Añadió que, mientras que en esta estructura en la que el mundo de lo público se alimenta en todos los sentidos de la esfera de lo privado, las mujeres nunca estarán bien, ni en paz, ni a gusto, ni realizadas: estarán extenuadas y fatigadas.

En cuanto al tema de la libre elección, Ana de Miguel señaló que no se pueden plantear en esos términos los comportamientos y la dinámica de la sociedad ni del ser humano. Desde el punto de vista de la investigación filosófica y sociológica, lo realmente importante, en su opinión, son las razones que determinan las elecciones de las mujeres. Dicho de otra manera, interesa estudiar las estructuras que determinan las elecciones libres de los hombres.

Para concluir, expuso que las mujeres han mejorado mucho, aunque sea a nivel de autoconciencia, reconociendo que no tendría sentido este Congreso si este fuera el mundo con el que las mujeres estuviesen conformes. En su opinión, las mujeres del sector público con seguridad en su empleo son las que deben pensar esto y proyectarlo a todos los demás en este mundo que no es el que se quiere y se debe cambiar.

A continuación, tuvo lugar la Mesa de Experiencias titulada "Instrumentos para la Igualdad", moderada por Pilar Madrid Yagüe, Presidenta de la Comisión de Igualdad de la Federación de Cuerpos Superiores de la Administración Civil del Estado (Fedeca), con el objeto de analizar cuestiones prácticas sobre algunas importantes herramientas para el avance en materia de género.

Desde el solemne y épico escenario de la Sala de Recepciones de la Excma. Diputación de Castellón, Pilar Batet Jiménez, miembro de la Comisión de Igualdad de la Diputación, y Ana Portal, Jefa de la recién creada Sección de Igualdad de esta Administración, "I Plan de Igualdad de la Diputación de Castellón”, expusieron, respectivamente, el Convenio 
suscrito con la Asociación de Mujeres en el Sector Público y el proceso de elaboración de dicho plan. Previamente se emitió un vídeo sobre el impulso que en materia de igualdad se está dando institucionalmente en Castellón.

Se señaló que al inicio del proceso de elaboración en 2019 se buscó una empresa consultora externa para que realizara el proceso de acompañamiento en el itinerario del plan, considerando que se obtendrían experiencias de buenas prácticas de otras empresas, lo que así sucedió. El Plan, aprobado en noviembre de 2020 y con cuatro años de vigencia, cuenta con 13 ejes y 63 acciones, "es el motor para poner en marcha ayudas tractoras en los Municipios más pequeños (subvenciones) para elaborar su propio plan de igualdad". Ana Portal concluyó señalando que "su evaluación dirá si el espejismo de la igualdad está realmente alcanzado".

Buenaventura Aguilera Díaz, Coordinador del Área de Estrategia en la Secretaría General de Hacienda de la Consejería de Hacienda y Financiación Europea de la Junta de Andalucía, "Presupuestos de género en Andalucía", destacó el liderazgo permanente de la Junta de Andalucía en esta herramienta a lo largo de 15 años, fruto del esfuerzo del personal del sector público andaluz y el apoyo político. En el año 2003, la Junta de Andalucía inició una estrategia de integración transversal del enfoque de género en las políticas generales a través del presupuesto público como vía necesaria a las políticas específicas de igualdad, tanto en la vertiente de los ingresos como en la de los gastos. Con un amplio anclaje normativo, la competencia está asignada al área de la Consejería de Hacienda y está dotada de estructura institucional. Además, señaló, se ha desarrollado una metodología presupuestaria basada en la clasificación de programas (Escala G+ a G0). El Ponente llamó especialmente la atención sobre el necesario acompañamiento de un Informe de Evaluación de Impacto de Género, informe muy relevante, afirmó, "puesto que el presupuesto no sería admitido en el Parlamento sin el mismo".

Consuelo Sánchez Naranjo, Administradora Civil del Estado y Asesora en el Gabinete de la Subsecretaría del Ministerio de Cultura y Deporte, "Observatorio de Igualdad de Género en el ámbito de la Cultura", expuso que el nacimiento de este Observatorio había surgido como "virtud de una necesidad", a la luz de los datos arrojados por las estadísticas oficiales en el curso académico 2017/18. En 2018 se puso en marcha una Comisión de Igualdad por parte del Ministerio de Cultura y Deporte y en febrero de 2019 se creó el Observatorio. En un informe elaborado por éste, afirmó, se evidencia que existe techo de cristal y cuesta mucho a las mujeres acceder a posiciones directivas. Existen datos abrumadores de mayoría de mujeres matriculadas en enseñanzas artísticas y universitarias relacionadas con profesiones culturales y, sin embargo, en 2019 las mujeres solo representaron el 40\% del "empleo cultural" (en media anual) y el acceso a puestos directivos fue testimonial.

Patricia Nieto Rojas, Profesora doctora en la Universidad Carlos III de Madrid, " $E x$ periencia de Igualdad de la Universidad Carlos III de Madrid", expuso el proceso de elaboración del II Plan de Igualdad y las diferencias con respecto al I Plan. A diferencia de este último, que fue efectuado por una empresa externa, para la elaboración del II Plan se nombró una Comisión interna formada por representantes de todos los colectivos de la Comunidad Universitaria: el personal de administración y de servicios, muy feminizado; 
el personal docente e investigador, con una mayor brecha de género; y el alumnado. A dichos colectivos se sumaron los sindicatos y el equipo de Gobierno. La Comisión arrancó sus trabajos en abril de 2016 y el II Plan se aprobó por Acuerdo del Consejo de Gobierno el 30 de octubre de 2017, con un período de vigencia de cinco años. Se trató de implicar al más alto nivel, haciendo responsable del mismo a los Vicerrectorados y Departamentos.

La Profesora Nieto Rojas subrayó que la mayor fortaleza de este II Plan fue su carácter omnicomprensivo, al incluir a la totalidad de los colectivos de la Comunidad Universitaria, y afirmó que estas medidas "harán una sociedad más igualitaria y, por tanto, una sociedad más competitiva”. Otras fortalezas en materia de género de esta Universidad son: el II Protocolo frente al acoso sexual y por razón de sexo y la inclusión en el Protocolo de Prevención del ciberacoso y la discriminación por orientación sexual.

María Amor Sánchez de Ron, Administradora Civil del Estado y Subdirectora Adjunta de Aprendizaje en el Instituto Nacional de Administración Pública (INAP), "Plan de formación en materia de género del INAP", expuso el I Plan de Formación en igualdad de género y no discriminación de este Instituto, aprobado en junio de 2020 para el período 2020-2022, con vocación de gran alcance (llegar no solo a la AGE, sino también a Comunidades Autónomas, Entidades Locales y otros organismos públicos) y aspirar al mayor impacto (alcanzar al mayor número de personas posible). Resaltó la apuesta del INAP por la innovación en la metodología, empleando nuevos recursos digitales, basados en la interactividad y colaboración entre participantes, e invitó a asistir a la presentación de la actividad formativa sobre prevención de violencia contra las mujeres que el INAP va a convocar próximamente.

A continuación, Carmen Seisdedos Alonso, Vicepresidenta de la Asociación de $\mathrm{Mu}$ jeres en el Sector Público, tomó la palabra para agradecer a las personas intervinientes su participación y dar paso a la clausura del Congreso.

María Vázquez Sellán, Subdirectora General de Estudios y Cooperación del Instituto de la Mujer y para la Igualdad de Oportunidades, Clausura del II Congreso de Mujeres en el Sector Público, comenzó trasladando un saludo en nombre de Beatriz Gimeno, Directora de este Instituto. Hizo un repaso del camino recorrido por las mujeres en su incorporación a las Administraciones Públicas y afirmó que, catorce años después de la Ley Orgánica 3/2007, sigue existiendo suelo pegajoso, techo de cristal y, además, segregación horizontal y vertical en el empleo público.

Señaló que es importante que en la Administración Pública tenga un papel fundamental el logro de la igualdad efectiva de las mujeres y provocar un efecto ejemplarizante sobre el empleo privado, "que sea un espejo para toda la sociedad". Y, mientras este momento llega, asociaciones como Mujeres en el Sector Público son fundamentales para "crear corrientes de sororidad entre todas nosotras".

Finalizó dando la enhorabuena a la Asociación por la iniciativa y el trabajo realizado en los dos años recorridos desde su creación, que "ha puesto el listón muy alto, pero los retos se nos dan muy bien a las mujeres", concluyó. 